\title{
ANO1 protein as a potential biomarker for esophageal cancer prognosis and precancerous lesion development prediction
}

\author{
Li Shang ${ }^{1, *}$, Jia-Jie Hao ${ }^{1, *}$, Xue-Ke Zhao ${ }^{3, *}$, Jian-Zhong He ${ }^{4, *}$, Zhi-Zhou Shi ${ }^{1}$, Hui- \\ Juan Liu ${ }^{5}$, Li-Fei Wu ${ }^{6}$, Yan-Yi Jiang ${ }^{1}$, Feng Shi ${ }^{1}$, Hai Yang ${ }^{1}$, Yu Zhang ${ }^{1}$, Yi-Zhen Liu ${ }^{1}$, \\ Tong-Tong Zhang ${ }^{1}$, Xin Xu${ }^{1}$, Yan Cai ${ }^{1}$, Xue-Mei Jia ${ }^{5}$, Min Li ${ }^{6}$, Qi-Min Zhan ${ }^{1}$, En-Min \\ $\mathbf{L i}^{4}$, Li-Dong Wang ${ }^{3}$, Wen-Qiang Wei ${ }^{2}$, Ming-Rong Wang ${ }^{1}$ \\ ${ }^{1}$ State Key Laboratory of Molecular Oncology, Cancer Institute (Hospital), Peking Union Medical College and Chinese Academy \\ of Medical Sciences, Beijing 100021, China \\ ${ }^{2}$ Department of Cancer Epidemiology, Cancer Institute (Hospital), Peking Union Medical College and Chinese Academy of \\ Medical Sciences, Beijing 100021, China \\ ${ }^{3}$ Henan Key Laboratory for Esophageal Cancer Research of the First Affiliated Hospital, Zhengzhou University, Zhengzhou \\ 450000, China \\ ${ }^{4}$ Department of Biochemistry and Molecular Biology, Shantou University Medical College, Shantou 515041, China \\ ${ }^{5}$ Department of Histology and Embryology, Anhui Medical University, Hefei 230032, China \\ ${ }^{6}$ Department of Gastroenterology, Anqing City Hospital, Affiliated Anqing Hospital of Anhui Medical University, Anqing \\ 246003, China \\ *These authors have contributed equally to this work \\ Correspondence to: Ming-Rong Wang, e-mail: wangmr2015@126.com \\ Wen-Qiang Wei, e-mail: weiwq@cicams.ac.cn \\ Li-Dong Wang, e-mail: ldwang2007@126.com \\ En-Min Li, e-mail: nmli@stu.edu.cn
}

Keywords: ANO1, esophageal squamous cell carcinoma, precancerous lesions, biomarker, prognosis

Received: September 17,2015 Accepted: March 01, $2016 \quad$ Published: March 21, 2016

\section{ABSTRACT}

Objectives: Anoctamin 1 (ANO1) has been found to be overexpressed in esophageal squamous cell carcinoma (ESCC) in our previous study. Herein we showed the clinical relevance of ANO1 alterations with ESCC and esophageal precancerous lesion progression.

Results: ANO1 was detected in 38.1\% (109/286) and 25.4\% $(77 / 303)$ of tumors in the two cohorts, but in none of morphologically normal operative margin tissues. ANO1 expression was significantly associated with a shorter overall survival (OS), especially in patients with moderately differentiated and stage IIA tumors. In 499 iodine-unstained biopsies from the endoscopic screening cohort in 2005-2007, all the 72 pathologically normal epithelial mucosa presented negative immunostaining, whereas ANO1 expression was observed in 3/11 tumors and 5/231 intraepithelial lesions. 7/8 ANO1-positive cases had developed unfavorable outcomes revealed by endoscopic follow-up in 2012. Analysis of another independent cohort of 148 intraepithelial lesions further confirmed the correlation between ANO1 expression and progression of precancerous lesions. 3/4 intraepithelial lesions with ANO1 expression had developed ESCC within 4-9 years after the initial endoscopic examination.

Methods: Immunohistochemistry (IHC) was performed to examine ANO1 expression in surgical ESCC specimens and two independent cohorts of esophageal biopsies from endoscopic screening in high-incidence area of ESCC in northern China. Association between ANO1 expression, clinico-pathologic parameters, and the impact on overall survival was analyzed.

Conclusions: Positive ANO1 is a promising biomarker to predict the unfavorable outcome for ESCC patients. More importantly, it can predict disease progression of precancerous lesions. 


\section{INTRODUCTION}

Esophageal carcinoma is one of the most common cancers. In China, squamous cell carcinoma (ESCC) is the predominant histological type of esophageal cancer. At the time of diagnosis, more than 50 percent of ESCC patients have unresectable tumors or radiographically visible metastases, and the overall five-year survival rate hovers around $10 \%$ for many years $[1,2]$. Accurate diagnosis and classification are of vital importance for improving clinical outcomes. But up to date, no powerful markers have been identified to accurately classifying ESCC individuals in conjunction with therapeutics. Another gray area of clinical management involves precancerous lesions of the esophagus, which is divided into low-grade intraepithelial lesions (LSIL) and high-grade intraepithelial lesions (HSIL). LSIL includes mild dysplasia $(\mathrm{mD})$ and moderate dysplasia (MD), and HSIL refers to severe dysplasia (SD) and carcinoma in situ (CIS). The clinical treatment for confirmed SD is usually the endoscopic mucosal resection, which is the same as that for CIS, but $\mathrm{mD}$ and $\mathrm{MD}$ are usually only regular follow-up [3]. Such management for precancerous lesions does not take into account the complex pattern of clonal evolution, which might lead to insufficient or excessive treatments. Identification of effective biomarkers that could be used to predict the prognosis of ESCC and the risk of precancerous lesions would be of great clinical benefits.

Anoctamin 1 (ANO1) has been recently identified to be overexpressed in esophageal carcinomas [4-7]. ANO1, also known as TMEM16A, ORAOV2, TAOS2, DOG1 or FLJ10261, is located on human chromosome 11q13. It contains 26 exons, encoding a 960 amino acid protein with eight transmembrane domains. ANO1 is associated with calcium-dependent chloride channel activity and involved in multiple biological functions including cell proliferation, motility and attachment $[8,9]$. It has been shown that ANO1 contributes to the regulation of renal function, inflammatory and nerve-injury induced hypersensitivity [10]. Amplification and/or overexpression of ANO1 have been frequently observed in gastrointestinal stromal tumors (GISTs) [11-15], breast cancer, head and neck squamous cell carcinoma (HNSCCs) and gastric carcinomas $[6,8,16]$ In ANO1-amplified cancer cell lines bearing 11q13 amplification, knockdown of ANO1 inhibited cell proliferation, induced apoptosis, and reduced tumor growth in established cancer xenografts via deactivating EGFR and CAMK signaling. Although a significant correlation has been found between ANO1 expression levels and overall survival (OS) of breast cancer patients [6], the clinical implication of ANO1 in human malignancies remain to be elucidated.

We previously found that ANO1 was amplified and overexpressed in both ESCC and esophageal dysplasia [7]. To further evaluate the clinical relevance of ANO1 alteration, we performed immunohistochemistry (IHC) to examine the expression of ANO1 protein not only in ESCCs who had undergone curative esophagectomy, but also in two independent biopsy cohorts of precancerous lesions to which we carried out an endoscopic follow-up for 4-9 years after their initial endoscopic examination.

\section{RESULTS}

\section{ANO1 expression in ESCC tissues and association with clinico-pathological features}

In the surgical specimens investigated, positive ANO1 staining was detected in $38.1 \%$ $(109 / 286)$ of tumors from the first cohort, but in none of all the morphologically normal operative margin tissues (Supplementary Figure S1). ANO1 expression was seen in the cytoplasm and cell membrane of the tumor cells, and was significantly associated with the location of tumors $(P=0.0201$, Supplementary Table S1). In the 286 tumors tested from the first cohort, the patient follow-up information of 162 cases was obtained, with an OS time of one to 168 months. Kaplan-Meier curves showed that patients with positive ANO1 expression had a shorter OS compared to those with undetectable expression $(P=0.0015$, Figure 1). Univariate Cox proportional hazards regression analysis showed that lymph node metastasis, advanced tumor stages and ANO1 expression were significantly correlated with poorer OS $(P=0.0012,0.0438$ and 0.0018). Multiple Cox proportional hazards regression analysis indicated that lymph node metastasis and ANO1 expression were independent prognostic factors for ESCC patients (hazard ratio $=1.8234$ and $1.9031, P=0.0146$ and 0.0020 , Table 1).

Stratified analysis in different clinico-pathological characteristics also indicated a poorer prognosis of ANO1-expressed patients, especially which were female $(P=0.0165)$, male $(P=0.0212)$, over 60 years old $(P=0.0008)$, and for those with tumors of middle location $(P=0.0066)$, moderate/poor differentiation (G2/3, $P=0.0047$ and 0.0256 , respectively), advanced stages (pT3 and stages IIB/III, $P=0.0035,0.0091$ and 0.0337 , respectively), as well as without lymph node metastasis and with N2 $(P=0.0389$ and 0.0096$)$ (Table 2, Figure 2).

Further stratified analysis was performed using another independent cohort from the second cohort and ANO1 were positively expressed in $25.4 \%$ (77/303) of patients. Also, all the morphologically normal operative margin tissues in this cohort showed ANO1 negative expression, which was the same as that in the first cohort. Although Kaplan-Meier curves showed no significant correlation between ANO1 expression and the OS of patients from the second cohort $(P=0.1615)$, patients 
with moderately differentiated and stage IIA tumors could be significantly stratified by ANO1 expression $(P=0.0453$ and 0.0091$)$ in the second cohort. Besides, male patients in both cohorts were stratified by ANO1 expression, although only marginal associations was observed in the second cohort $(P=0.0698)$ (Table 2, Figure 2).

\section{ANO1 expression in endoscopic biopsies in high- incidence areas}

In the first cohort of biopsy specimens, ANO1 expression was detected in 3 out of 11 pathologically confirmed squamous carcinomas. Of the 416 cases with precancerous lesions, five cases presented positive ANO1 staining, including two cases with $\mathrm{mD}$, one with $\mathrm{MD}$, one with SD, and one with CIS (Figure 3A). However, all the 72 pathologically normal epithelial mucosa showed undetectable ANO1 immunoreaction. In the second cohort, ANO1 expression was observed in three of four cases with
SD and one with CIS (Figure 3B), but undetectable in all the $43 \mathrm{mD}, 6 \mathrm{MD}$ and 94 chronic esophagitis.

\section{Progression of esophageal precancerous lesions with positive ANO1 expression in two independent cohorts from endoscopy screening}

Endoscopic follow-up was carried out for 4-9 years after initial biopsy examination. The follow-up data of the two independent cohorts were summarized in Supplementary Tables S2-S3. We observed the progression of the cases with positive ANO1 expression. In the first cohort, all the three patients with squamous carcinomas had died at the follow-up time, those with SD or CIS developed squamous carcinomas, the case with MD progressed to intraepithelial carcinoma, one of the two $\mathrm{mD}$ progressed to $\mathrm{MD}$ and another regressed to a mild esophagitis. And in the second cohort, patients with CIS and two with SD had developed squamous carcinomas, and another SD remained unchanged (Table 3). Taken together, the precancerous lesions with

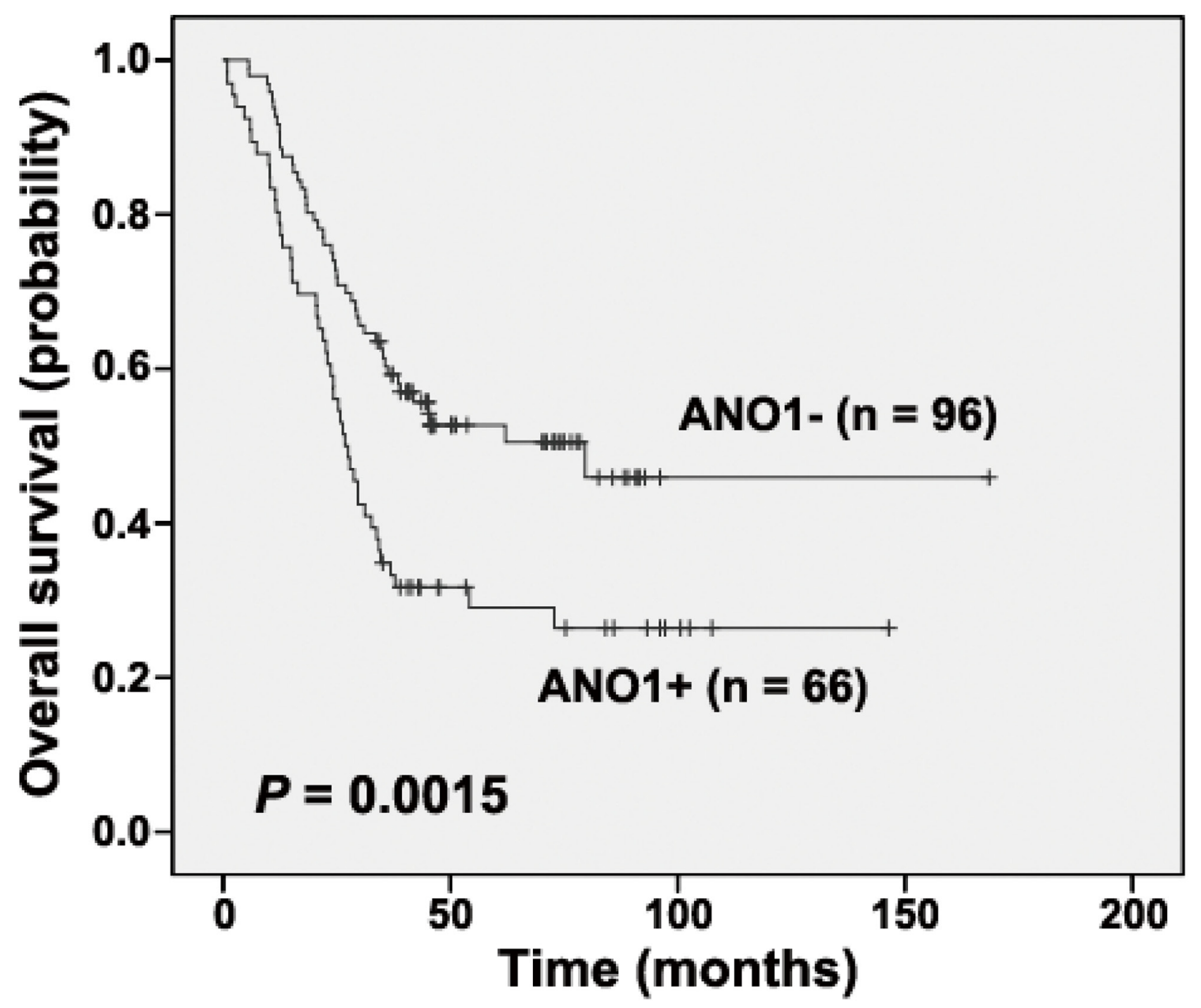

Figure 1: Kaplan-Meier analysis according to ANO1 status in cases from the first cohort (Log-rank test). “+”, positive; “-”, negative. 
Table 1: Univariate and multivariate cox regression analysis of overall survival of ESCC patients in CAMS cohort

\begin{tabular}{|c|c|c|c|c|c|c|}
\hline \multirow[t]{2}{*}{ Variable } & \multicolumn{3}{|c|}{ Univariate analysis } & \multicolumn{3}{|c|}{ Multivariate analysis } \\
\hline & HR & $95 \% \mathrm{CI}$ & $P$ value & HR & $95 \% \mathrm{CI}$ & $P$ value \\
\hline Sex (Female, Male) & 1.2533 & $\begin{array}{c}0.7767- \\
2.0222\end{array}$ & 0.3550 & & & \\
\hline Age $(\leq 60,>60)$ & 0.6990 & $\begin{array}{c}0.4635- \\
1.0542\end{array}$ & 0.0876 & & & \\
\hline Macroscopic types (Ul, Fu, Me) & 1.0699 & $\begin{array}{c}0.7665- \\
1.4935\end{array}$ & 0.6912 & & & \\
\hline Tumor location (Up, Mi, Lo) & 0.9403 & $\begin{array}{c}0.6827- \\
1.2950\end{array}$ & 0.7061 & & & \\
\hline Tumor size $(\leq 5,>5)$ & 2.3524 & $\begin{array}{c}0.8957- \\
2.0419\end{array}$ & 0.1510 & & & \\
\hline pT (T1-2, T3-4) & 1.3584 & $\begin{array}{c}0.7046- \\
2.6187\end{array}$ & 0.3604 & & & \\
\hline $\mathbf{p N}(\mathrm{N} 0, \mathrm{~N} 1-3)$ & 2.0212 & $\begin{array}{c}1.3201- \\
3.0947\end{array}$ & 0.0012 & 1.8234 & $\begin{array}{l}1.1259- \\
2.9528\end{array}$ & 0.0146 \\
\hline Grade (G1, G2, G3) & 1.0777 & $\begin{array}{c}0.7912- \\
1.4679\end{array}$ & 0.6352 & & & \\
\hline Stage (I/IIA, IIB/IIIC) & 1.9646 & $\begin{array}{c}1.0188- \\
3.7884\end{array}$ & 0.0438 & 1.3322 & $\begin{array}{c}0.6337- \\
2.8008\end{array}$ & 0.4493 \\
\hline ANO1 (Neg, Pos) & 1.9141 & $\begin{array}{l}1.2729- \\
2.8782\end{array}$ & 0.0018 & 1.9031 & $\begin{array}{l}1.2653- \\
2.8624\end{array}$ & 0.0020 \\
\hline
\end{tabular}

HR, hazard ratio; CI, confidence interval; Ul, Ulcerative; Fu, Fungating; Me, Medullary; Up, Upper; Mi, Middle; Lo, Lower; pN, lymph node metastases; Neg: negative expression; Pos: positive expression.

Table 2: Stratified analysis of ANO1 expression for overall survival in ESCC patients in two independent cohorts

\begin{tabular}{|c|c|c|c|c|}
\hline \multirow[t]{2}{*}{ Parameter } & \multicolumn{2}{|c|}{ Cohort $1^{\#}$} & \multicolumn{2}{|c|}{ Cohort $2^{\#}$} \\
\hline & $\mathbf{n}$ & $P$ value & $\mathbf{n}$ & $P$ value \\
\hline \multicolumn{5}{|l|}{ Sex } \\
\hline Female & 42 & 0.0165 & 66 & 0.0914 \\
\hline Male & 120 & 0.0212 & 237 & 0.0698 \\
\hline \multicolumn{5}{|l|}{ Age } \\
\hline$\leq 60$ & 85 & 0.2417 & 190 & 0.9951 \\
\hline$>60$ & 77 & 0.0008 & 113 & 0.1265 \\
\hline \multicolumn{5}{|c|}{ Tumor location } \\
\hline Upper & 28 & 0.5970 & 18 & 0.5252 \\
\hline Middle & 94 & 0.0066 & 124 & 0.4682 \\
\hline Lower & 40 & 0.0544 & 161 & 0.3878 \\
\hline \multicolumn{5}{|l|}{ pT } \\
\hline pT1 & - & - & - & - \\
\hline pT2 & 19 & 0.2484 & 48 & 0.1203 \\
\hline pT3 & 111 & 0.0035 & 242 & 0.1652 \\
\hline pT4 & 31 & 0.0623 & - & - \\
\hline
\end{tabular}




\begin{tabular}{cccccc}
\hline Parameter & \multicolumn{2}{c}{ Cohort 1 $^{\#}$} & & \multicolumn{3}{c}{ Cohort 2 $^{\#}$} \\
\cline { 2 - 3 } \cline { 5 - 6 } Grade & $\mathbf{n}$ & $\boldsymbol{P}_{\text {value }}$ & $\mathbf{n}$ & $\boldsymbol{P}_{\text {value }}$ \\
G1 & 33 & & & & \\
G2 & 87 & 0.8028 & & 47 & 0.4760 \\
G3 & 42 & 0.0047 & & 231 & 0.0453 \\
pN & & 0.0256 & & 25 & 0.3625 \\
N0 & 76 & 0.0389 & & 149 & \\
N1 & 43 & 0.5980 & & 83 & 0.1376 \\
N2 & 31 & 0.0096 & & 52 & 0.8570 \\
N3 & 12 & 0.7822 & & 19 & 0.6440 \\
Stage & & & & 0.9773 \\
I & 11 & 0.3112 & & 29 & 0.7699 \\
IIA & 15 & 0.4450 & & 76 & 0.0091 \\
IIB & 41 & 0.0091 & & 60 & 0.3088 \\
III & 95 & 0.0337 & & 138 & 0.9606 \\
\hline
\end{tabular}

pT, pathologic T stage; $\mathrm{pN}$, lymph node metastases.
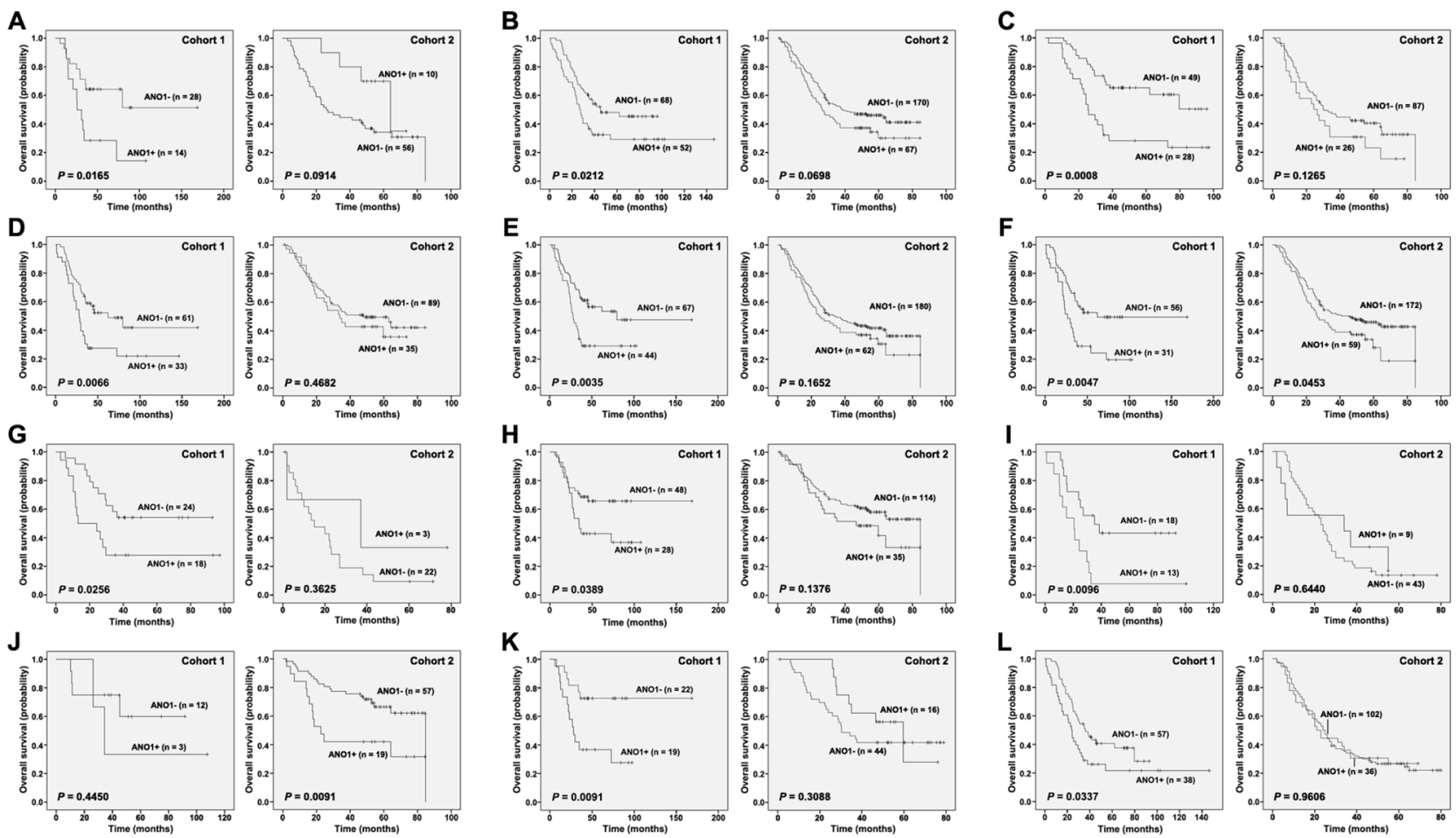

Figure 2: Stratified Kaplan-Meier analysis according to ANO1 status in two independent cohorts (Log-rank test). A. Female; B. Male; C. Age (> 60 years old); D. middle location; E. pT3; F. Grade 2; G. Grade 3; H. pN0; I. pN2; J. Stage IIA; K. Stage IIB; L. Stage III. “+”, positive; “-”, negative. 
A

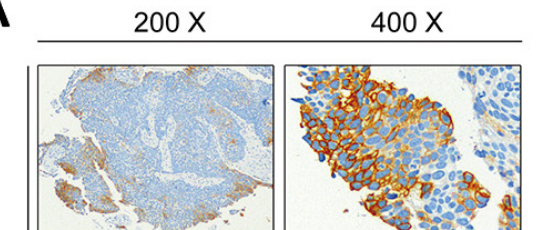

\begin{tabular}{l|l}
0 \\
W
\end{tabular}

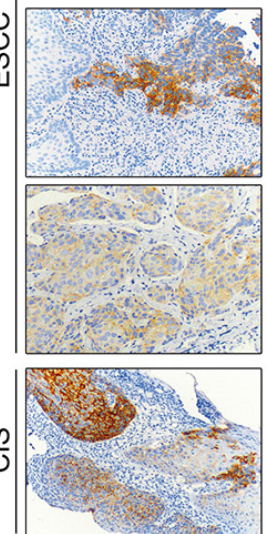

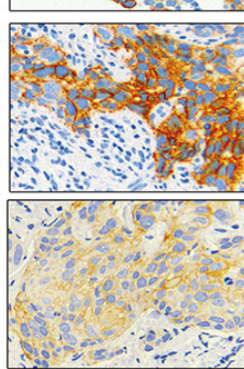

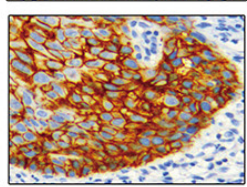

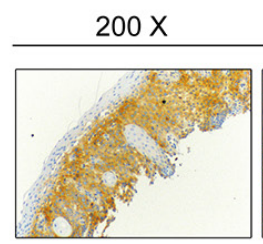
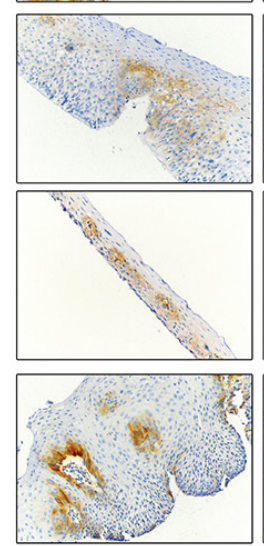

$400 X$

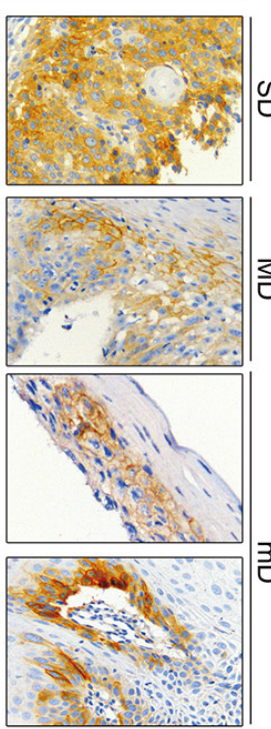

B

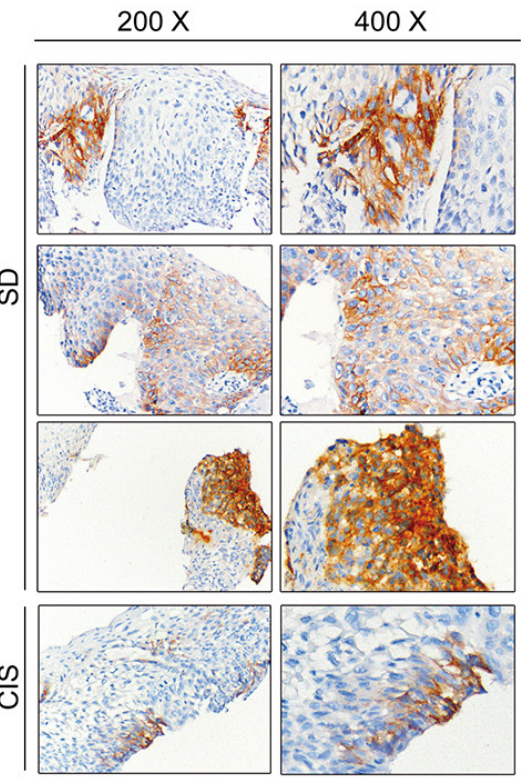

Figure 3. Representative immunohistochemical results of ANO1 positive staining. A. Positive immunohistochemical staining of ANO1 in precancerous lesions and ESCC tissues in the sample set between 2005 and 2007 (magnification 200× and 400×). B. Positive immunohistochemical staining of ANO1 in precancerous lesions in the sample set between 1996 and 2002 (magnification 200× and 400×). $\mathrm{mD}$, mild dysplasia; MD, moderate dysplasia; SD, severe hyperplasia; CIS, carcinoma in situ; ESCC, esophageal squamous cell carcinoma.

Table 3: Progression of esophageal lesions with positive ANO1 expression in two independent cohorts from endoscopy screening

\begin{tabular}{lccccc}
\hline Cohort & $\begin{array}{c}\text { Initial } \\
\text { examination }\end{array}$ & Initial diagnosis & Endpoint Time & Endpoint Result & Alteration \\
\hline 1 st & 2007 & ESCC & 2011 & Died of ESCC & Progressed \\
1 st & 2007 & ESCC & 2012 & Died of ESCC & Progressed \\
1 st & 2007 & ESCC & 2010 & Died of ESCC & Progressed \\
1 st & 2006 & CIS & 2006 & ESCC diagnosed & Progressed \\
1 st & 2007 & SD & 2012 & ESCC diagnosed & Progressed \\
1 st & 2005 & MD & 2009 & Intramucosal & Progressed \\
1 carcinoma & MD diagnosed & Progressed \\
1st & 2007 & mD & 2012 & ES diagnosed & Regressed \\
2nd & mD & 2012 & ESCC diagnosed & Progressed \\
2nd & 2005 & CIS & 2005 & ESCC diagnosed & Progressed \\
2nd & SD & SD & 2000 & Died of ESCC & Progressed \\
2nd & 1998 & SD & 2005 & SD diagnosed & Unchanged \\
\hline
\end{tabular}

ES, esophagitis; mD, mild dysplasia; MD, moderate dysplasia; SD, severe hyperplasia; CIS, carcinoma in situ;

ESCC, esophageal squamous cell carcinoma. 
positive ANO1 expression mostly had progressed in both the two cohort ( $80 \%$ and $100 \%$ ), and the vast majority of those with negative ANO1 expression were no progression $(87.8 \%$ and $100 \%$ ) (Supplementary Table S4).

\section{DISCUSSION}

In the present study, we demonstrated that ANO1 expression was an independent prognostic biomarker for shorter survival of ESCC patients. More importantly, we showed for the first time that ANO1 expression was a potential biomarker for predicting disease progression in subjects with esophageal precancerous lesions.

We observed that ANO1 expression was positive in $38.1 \%(109 / 286)$ and $25.4 \%(77 / 303)$ of primary esophageal tumors from the two independent cohorts, respectively. Excitingly, all the morphologically normal operative margin tissues from both cohorts showed negative ANO1 expression. Also, in the endoscopic biopsies, all the pathologically normal epithelial mucosa and chronic esophagitis showed undetectable ANO1 immunoreaction. These results suggest a high specificity (100\%) of ANO1 expression.

We found a correlation between ANO1 expression and shorter survival time of patients. Multiple Cox regression identified that ANO1 expression was an independent prognostic factor for shorter survival. When comparing the stratified analysis results from two independent cohorts, we found that ANO1-expressed patients with moderately differentiated tumors showed poorer prognosis in both cohorts. It is noted that two different prognosis groups could also be separated according to Kaplan-Meier curves when stratification analysis was performed on patients that were male and over 60 years old, and those with pT3 and pN0 tumors, although the differences were not significant in the second cohort. Besides, stage IIA patients were stratified by ANO1 expression in the second cohort but not in the first cohort. Whereas middle location, poor differentiation, $\mathrm{pN} 2$, stages IIB/III could significantly stratify patients in the first cohort but not in the second cohort. The possible reason for the discrepancies among different cohorts is that the patterns of other molecular alterations may be variable among different areas, in which populations, environments and carcinogenic factors may be different from each other. Whether it is caused by the area differences should be further validated. But importantly, we noted that in tumors of ANO1 immunostaining score $\geq 6$ in the present study, $53.1 \%(26 / 49)$ had died up to the follow-up time, in which primary tumors of eleven patients were at stages I-II. These data suggest that ANO1 expression could indicate a subset of patients for whom more aggressive treatments and close follow-up should be considered.

Based on that ANO1 expression presented 100\% specificity in morphologically normal operative margin tissues and its prognostic implication in patients with advanced ESCC, we further investigated ANO1 alteration in esophageal precancerous lesions according to the follow-up information. We observed that $80 \%$ (four out of five) of ANO1-positive cases detected in initial biopsies showed lesion progression at the follow-up time in the first cohort, and $75 \%$ (three out of four) showed progression in the second cohort. Of these seven progressed cases, five patients had developed invasive squamous carcinomas, one progressed to intramucosal carcinoma, and one $\mathrm{mD}$ progressed to MD. The data indicate that ANO1 may be a potential biomarker for predicting malignant progression of precancerous lesions. In China, the overall survival rate of ESCC patients is less than $10 \%$, but as high as $85 \%$ if the patients are treated at an earlier stage [2, 17]. A previous study showed that endoscopic mucosal resection (EMR) had an excellent long-term survival rate of $97 \%$ with acceptable function and quality of life [18]. Therefore, the development of appropriate early detection and prediction biomarkers for curable lesions offer a great opportunity to reduce the risk of progressing to ESCC. Our results suggest that mucosal resection plus a strengthened follow-up might be essential for ANO1positive esophageal precancerous lesions, especially for those with strong ANO1 expression. In the future, we will extend the present study to a prospective investigation in order to confirm the potential clinical value of ANO1 for predicting the progression of precancerous lesions.

It has been revealed that ANO1-induced cancer cell proliferation was accompanied by an increase of extracellular signal-regulated kinase (ERK)1/2 activation and cyclin D1 induction in head and neck squamous cell carcinoma. Pharmacologic inhibition of MEK/ERK and genetic inactivation of ERK1/2 could abrogate the growth effect of ANO1 [19]. A similar ANO1-regulated ERK1/2 phsphorylation has been observed in mouse ovarian granulosa cells [20]. We previously reported that knockdown of ANO1 significantly inhibited the proliferation of KYSE30 and KYSE510 cells [7]. However, the mechanisms underlying the involvement of ANO1 in esophageal carcinogenesis remains unclear, to which further investigation should be addressed.

In conclusion, ANO1 expression can serve as a poorer prognosis biomarker for ESCC patients to whom a more aggressive treatment should be considered. Especially, our study provides an important candidate biomarker to predict disease progression in individuals with esophageal precancerous lesions.

\section{MATERIALS AND METHODS}

\section{Patients and samples}

A total of 589 surgically resected primary ESCC tissues of two independent cohorts from two different areas of China and 647 esophageal endoscopic biopsies (including 564 intraepithelial lesions) were used in the present study. 
286 ESCC tissues from the first cohort were collected between 1998 and 2008 at the Cancer Hospital, Chinese Academy of Medical Sciences and Peking Union Medical College (CAMS \& PUMC) in Beijing, China. 303 ESCC tissues from the second cohort were collected between 2007 and 2011 at Medical College of Shantou University in Shantou, China. All the operative samples were residual specimens after diagnostic sampling. All the patients received no treatment before surgery, and patients who died within one month after surgery were excluded. Clinico-pathological characteristics of the 589 ESCC patients were summarized in Supplementary Table S5.

The biopsy tissues included two independent cohorts. The first one contained 499 iodine-unstained biopsies collected by endoscopic screening from 2005 to 2007 in ESCC high-incidence area, Linxian and Cixian, China. Among them, 72 were pathologically diagnosed as normal epithelia, 11 as squamous carcinomas and 416 as precancerous lesions $(339 \mathrm{mD}, 54 \mathrm{MD}, 19 \mathrm{SD}$ and 4 carcinoma in situ). The second cohort included 94 chronic esophagitis tissues and 54 precancerous lesions $(43 \mathrm{mD}$, $6 \mathrm{MD}, 4 \mathrm{SD}$ and one carcinoma in situ) procured by endoscopic examination in another rural area of Linxian from 1996 to 2002. All the biopsy specimens for this study were collected as residual tissue sections after clinical diagnosis. Clinico-pathological characteristics of the $564(416+94+54)$ patients with esophageal precancerous lesions were summarized in Supplementary Table S6. For patients of the two cohorts, endoscopic follow-up was carried out in 2012 for the first cohort and 4-9 years after initial endoscopic examination for the second cohort.

Every patient signed separate informed consent forms for sampling and molecular analysis. Tissues were routinely formalin-fixed and paraffin-embedded.

This study was approved by the Ethics Committee/ Institutional Review Board of the Cancer Institute (Hospital), Peking Union Medical College and Chinese Academy of Medical Sciences (No. 12-097/631).

\section{Sample preparation and IHC procedure}

Tissue microarrays (TMAs) for advanced carcinomas were constructed as described previously [21], and the resulting blocks were cut into 4- $\mu \mathrm{m}$ sections. Biopsy tissues were cut into $4-\mu \mathrm{m}$ full sections. The slides were deparaffinized, rehydrated, immersed in $3 \%$ hydrogen peroxide solution for $10 \mathrm{~min}$, heated in citrate buffer ( $\mathrm{pH}$ 6.0) for $25 \mathrm{~min}$ at $95^{\circ} \mathrm{C}$, and cooled for 60 min at room temperature. Between each incubation step, the slides were washed with phosphate buffered saline (PBS, $\mathrm{pH}$ 7.4). Then the slides with TMAs were incubated with rabbit monoclonal antibody (1:50 dilution, LifeSpan BioSciences, Seattle, USA) overnight at 4 ${ }^{\circ} \mathrm{C}$. Immunostaining was performed using the PV-9000 Polymer Detection System with diaminobenzidine (DAB) according to manufacturer recommendations (GBI, USA) and subsequently counterstained with hematoxylin. Slides without the addition of primary antibody served as negative control.

\section{Assessment of IHC results}

Assessment and imaging of the IHC results were performed using a Leica DM2000 microscope equipped with Leica DFC Cameras-Image Acquisition System (software V3.5.0, Switzerland). Immunohistochemical staining was scored blindly with no information on the clinical data provided. ANO1 protein expression was determined based on staining intensity and the percentage of immunoreactive cells. The staining intensity was rated as 0 (no staining), 1 (weak staining), 2 (moderate staining), or 3 (strong staining). The percentage of immunoreactive cells was graded as $0(\leq$ $10 \%), 1(11-25 \%), 2(26-50 \%)$, or $3(>50 \%)$. Tissue IHC score was calculated by multiplying the intensity and the percentage of positive tumor cells.

\section{Statistical analysis}

Statistical analysis was performed with the SPSS software program (version 17.0). Chi square test was used to assess the relationship between ANO1 expression and clinico-pathological parameters. Kaplan-Meier survival curves were constructed, and the differences were detected by the Log-rank test. The clinical end point was OS, defined as time from surgery to death. The data of patients alive at the end of the study were censored. Multiple Cox proportional hazards regression was carried out to identify the independent factors with a significant impact on patient survival. $P$ values less than 0.05 were considered statistically significant.

\section{ACKNOWLEDGMENTS}

The authors would like to thank all members of the Wang MR lab for the critical discussion.

\section{CONFLICTS OF INTEREST}

None of the authors of this manuscript have any conflicts of interest related to this work.

\section{GRANT SUPPORT}

This work was supported by National High Technology Research and Development Program of China (2012AA02A503), National Natural Science Foundation of China (81330052, 81460425, 81520108023) and Natural Science Foundation of Beijing (7151008). 


\section{REFERENCES}

1. Enzinger PC and Mayer RJ. Esophageal cancer. The New England journal of medicine. 2003; 349:2241-2252.

2. Jemal A, Bray F, Center MM, Ferlay J, Ward E and Forman D. Global cancer statistics. CA Cancer J Clin. 2011; 61:69-90.

3. Allum WH, Blazeby JM, Griffin SM, Cunningham D, Jankowski JA and Wong R. Guidelines for the management of oesophageal and gastric cancer. Gut. 2011; 60:1449-1472.

4. Kashyap MK, Marimuthu A, Kishore CJ, Peri S, Keerthikumar S, Prasad TS, Mahmood R, Rao S, Ranganathan P, Sanjeeviah RC, Vijayakumar M, Kumar $\mathrm{KV}$, Montgomery EA, Kumar RV and Pandey A. Genomewide mRNA profiling of esophageal squamous cell carcinoma for identification of cancer biomarkers. Cancer biology \& therapy. 2009; 8:36-46.

5. Ruiz C, Martins JR, Rudin F, Schneider S, Dietsche T, Fischer CA, Tornillo L, Terracciano LM, Schreiber R, Bubendorf L and Kunzelmann K. Enhanced expression of ANO1 in head and neck squamous cell carcinoma causes cell migration and correlates with poor prognosis. PloS one. 2012; 7:e43265.

6. Britschgi A, Bill A, Brinkhaus H, Rothwell C, Clay I, Duss S, Rebhan M, Raman P, Guy CT, Wetzel K, George E, Popa MO, Lilley S, Choudhury H, Gosling M, Wang L, et al. Calcium-activated chloride channel ANO1 promotes breast cancer progression by activating EGFR and CAMK signaling. Proceedings of the National Academy of Sciences of the United States of America. 2013; 110:E1026-1034.

7. Shi ZZ, Shang L, Jiang YY, Hao JJ, Zhang Y, Zhang TT, Lin DC, Liu SG, Wang BS, Gong T, Zhan QM and Wang MR. Consistent and differential genetic aberrations between esophageal dysplasia and squamous cell carcinoma detected by array comparative genomic hybridization. Clin Cancer Res. 2013; 19:5867-5878.

8. Ayoub C, Wasylyk C, Li Y, Thomas E, Marisa L, Robe A, Roux M, Abecassis J, de Reynies A and Wasylyk B. ANO1 amplification and expression in HNSCC with a high propensity for future distant metastasis and its functions in HNSCC cell lines. British journal of cancer. 2010; 103:715-726.

9. Liu W, Lu M, Liu B, Huang Y and Wang K. Inhibition of $\mathrm{Ca}(2+)$-activated $\mathrm{Cl}(-)$ channel ANO1/TMEM16A expression suppresses tumor growth and invasiveness in human prostate carcinoma. Cancer letters. 2012; 326:41-51.

10. Lee B, Cho H, Jung J, Yang YD, Yang DJ and Oh U. Anoctamin 1 contributes to inflammatory and nerve-injury induced hypersensitivity. Molecular pain. 2014; 10:5.

11. West RB, Corless CL, Chen X, Rubin BP, Subramanian S, Montgomery K, Zhu S, Ball CA, Nielsen TO, Patel R, Goldblum JR, Brown PO, Heinrich MC and van de Rijn M. The novel marker, DOG1, is expressed ubiquitously in gastrointestinal stromal tumors irrespective of KIT or PDGFRA mutation status. The American journal of pathology. 2004; 165:107-113.

12. Espinosa I, Lee CH, Kim MK, Rouse BT, Subramanian S, Montgomery K, Varma S, Corless CL, Heinrich MC, Smith KS, Wang Z, Rubin B, Nielsen TO, Seitz RS, Ross DT, West RB, et al. A novel monoclonal antibody against DOG1 is a sensitive and specific marker for gastrointestinal stromal tumors. The American journal of surgical pathology. 2008; 32:210-218.

13. Miettinen M, Wang ZF and Lasota J. DOG1 antibody in the differential diagnosis of gastrointestinal stromal tumors: a study of 1840 cases. The American journal of surgical pathology. 2009; 33:1401-1408.

14. Lopes LF, West RB, Bacchi LM, van de Rijn M and Bacchi CE. DOG1 for the diagnosis of gastrointestinal stromal tumor (GIST): Comparison between 2 different antibodies. Appl Immunohistochem Mol Morphol. 2010; 18:333-337.

15. Akpalo $\mathrm{H}$, Lange $\mathrm{C}$ and Zustin J. Discovered on gastrointestinal stromal tumour 1 (DOG1): a useful immunohistochemical marker for diagnosing chondroblastoma. Histopathology. 2012; 60:1099-1106.

16. Yang J, Liu N, Kang A, Jin Y, Wang J, Su B, Chen X and Li Z. [Expression of TMEM16A in gastric carcinoma and its clinical implications]. Nan fang yi ke da xue xue bao= Journal of Southern Medical University. 2012; 32:794-797.

17. Wang GQ, Jiao GG, Chang FB, Fang WH, Song JX, Lu N, Lin DM, Xie YQ and Yang L. Long-term results of operation for 420 patients with early squamous cell esophageal carcinoma discovered by screening. The Annals of thoracic surgery. 2004; 77:1740-1744.

18. Wang GQ, Hao CQ, Wei WQ and Zhao L. [Long-term outcomes of endoscopic argon plasma coagulation (APC) therapy for early esophageal cancer and precancerous lesions]. Zhonghua zhong liu za zhi [Chinese journal of oncology]. 2013; 35:456-458.

19. Duvvuri U, Shiwarski DJ, Xiao D, Bertrand C, Huang X, Edinger RS, Rock JR, Harfe BD, Henson BJ, Kunzelmann K, Schreiber R, Seethala RS, Egloff AM, Chen X, Lui VW, Grandis JR, et al. TMEM16A induces MAPK and contributes directly to tumorigenesis and cancer progression. Cancer research. 2012; 72:3270-3281.

20. Sun M, Sui Y, Li L, Su W, Hao F, Zhu Q, Di W, Gao H and Ma T. Anoctamin 1 calcium-activated chloride channel downregulates estrogen production in mouse ovarian granulosa cells. Endocrinology. 2014; 155:2787-2796.

21. Feng YB, Lin DC, Shi ZZ, Wang XC, Shen XM, Zhang Y, Du XL, Luo ML, Xu X, Han YL, Cai Y, Zhang ZQ, Zhan QM and Wang MR. Overexpression of PLK1 is associated with poor survival by inhibiting apoptosis via enhancement of survivin level in esophageal squamous cell carcinoma. Int J Cancer. 2009; 124:578-588. 